\title{
Site of Action Potential Initiation in Layer 5 Pyramidal Neurons
}

\author{
Lucy M. Palmer and Greg J. Stuart \\ Division of Neuroscience, John Curtin School of Medical Research, Australian National University, Canberra 0200, Australia
}

Fundamental to an understanding of how neurons integrate synaptic input is the knowledge of where within a neuron this information is converted into an output signal, the action potential. Although it has been known for some time that action potential initiation occurs within the axon of neurons, the precise location has remained elusive. Here, we provide direct evidence using voltage-sensitive dyes that the site of action potential initiation in cortical layer 5 pyramidal neurons is $\sim 35 \mu \mathrm{m}$ from the axon hillock. This was the case during action potential generation under a variety of conditions, after axonal inhibition, and at different stages of development. Once initiated action potentials propagated down the axon in a saltatory manner. Experiments using local application of low-sodium solution and TTX, as well as an investigation of the influence of axonal length on action potential properties, provided evidence that the initial $40 \mu \mathrm{m}$ of the axon is essential for action potential generation. To morphologically identify the relationship between the site of action potential initiation and axonal myelination, we labeled oligodendrocytes supplying processes to the proximal region of the axon. These experiments indicated that the axon initial segment was $\sim 40 \mu \mathrm{m}$ in length, and the first node of Ranvier was $\sim 90 \mu \mathrm{m}$ from the axon hillock. Experiments targeting the first node of Ranvier suggested it was not involved in action potential initiation. In conclusion, these results indicate that, in layer 5 pyramidal neurons, action potentials are generated in the distal region of the axon initial segment.

Key words: cortex; axon; initial segment; myelin; oligodendrocyte; saltatory conduction

\section{Introduction}

Action potentials (APs) are the fundamental electrical signal used by the nervous system to relay information. In single neurons, information is carried by synaptic inputs via an increase or decrease in the probability of AP generation in a process called synaptic integration. Given that neurons usually receive thousands of synaptic inputs distributed across complex dendritic trees (extending up to $1 \mathrm{~mm}$ from the cell body), an essential step to understanding the process of synaptic integration is the knowledge of where within a neuron APs are generated. Pioneering work in spinal motoneurons in the 1950s suggested the AP is generated in the axon initial segment or possibly the first nodes of Ranvier (Coombs et al., 1957; Fatt, 1957; Fuortes et al., 1957). Subsequent studies have supported this idea in other neuronal types, with direct evidence for an axonal site of AP initiation coming from simultaneous axonal and somatic recordings in the 1990s (Stuart and Häusser, 1994; Stuart and Sakmann, 1994; Colbert and Johnston, 1996; Stuart et al., 1997a).

Based on the assumption that the axon initial segment contains a high density of voltage-activated sodium channels, theoretical studies predict AP initiation will occur in the axon initial segment (Dodge and Cooley, 1973; Mainen et al., 1995). Experimental evidence for a high density of sodium channels in the

\footnotetext{
Received Nov. 9, 2005; revised Dec. 22, 2005; accepted Dec. 22, 2005.

This work was supported by the Alexander von Humboldt Foundation, the John Curtin School of Medical Research, and a Fenner PhD scholarship to L.M.P. We thank John Clements for help with analysis.

Correspondence should be addressed to Greg J. Stuart at the above address. E-mail: greg.stuart@anu.edu.au. DOI:10.1523/JNEUROSCI.4812-05.2006

Copyright $\odot 2006$ Society for Neuroscience $\quad 0270-6474 / 06 / 261854-10 \$ 15.00 / 0$
}

axon initial segment is contradictory, however. Patch experiments detect little or no difference in the density of sodium channels in the initial segment compared with that found at the soma (Colbert and Johnston, 1996; Colbert and Pan, 2002; Ruben et al. 2003), whereas antibody studies suggest a high density of sodium channel staining in the axon initial segment in many neuronal types (Wollner and Catterall, 1986; Jenkins and Bennett, 2001; Komada and Soriano, 2002; Boiko et al., 2003). Alternatively, differences in sodium channel properties may underlie AP initiation in the absence of a high sodium channel density (Colbert and Pan, 2002). Consistent with a low density of sodium channels in the axon initial segment, there is evidence that AP initiation can occur at the first nodes of Ranvier in some neuronal types (Gogan et al., 1983; Colbert and Johnston, 1996; Clark et al., 2005).

In principle, it should be possible to address the question of where in the axon APs are initiated via intracellular recording at different axonal sites. However, because of the small diameter of the axon $(<1 \mu \mathrm{m})$, as well as myelination, this is beyond the scope of current recording techniques. Moreover, to localize the site of AP initiation based on small differences in AP latency, one would ideally need to record simultaneously from multiple axonal sites. Here, we address the issue of the site of AP initiation in cortical layer 5 pyramidal neurons using the intracellular application of voltage-sensitive dyes: a method that allows the simultaneous monitoring of axonal membrane potential at multiple sites. The results provide direct experimental evidence that, in layer 5 pyramidal neurons, AP initiation occurs in the distal region of the axon initial segment. 


\section{Materials and Methods}

Wistar rats (3-5 weeks of age, unless otherwise stated) were anesthetized by inhalation of halothane and decapitated according to the Animal Experimentation Ethics Committee of the Australian National University. Somatosensory neocortical brain slices (300 $\mu \mathrm{m}$ thick) were prepared using a vibrating tissue slicer (Dosaka, Kyoto, Japan) and perfused with oxygenated $\left(95 \% \mathrm{O}_{2} / 5 \% \mathrm{CO}_{2}\right)$ extracellular solution containing the following (in mM): $125 \mathrm{NaCl}, 25 \mathrm{NaHCO}_{3}, 25$ glucose, $3 \mathrm{KCl}, 1.25$ $\mathrm{NaH}_{2} \mathrm{PO}_{4}, 2 \mathrm{CaCl}_{2}, 1 \mathrm{MgCl}_{2}$, pH 7.4 (osmolarity, 300-310 mOsm/L). Slices were incubated at $35^{\circ} \mathrm{C}$ for $30 \mathrm{~min}$ and subsequently maintained at room temperature $\left(\sim 22^{\circ} \mathrm{C}\right)$ until required. Somatic whole-cell recordings were made from visually identified layer 5 pyramidal neurons using infrared differential interference contrast (DIC) optics (Stuart et al., 1993) and intracellular and extracellular recordings made with currentclamp amplifiers (Dagan, Minneapolis, MN). Whole-cell recording pipettes (5-7 M $\Omega$ ) were filled with $135 \mathrm{~mm} \mathrm{~K}$-gluconate, $7 \mathrm{~mm} \mathrm{NaCl}, 10 \mathrm{mM}$ HEPES, $2 \mathrm{~mm} \mathrm{MgCl}_{2}, 2 \mathrm{~mm} \mathrm{Na}_{2}$-ATP, and $0.3 \mathrm{~mm} \mathrm{Na}_{2}$-GTP (pH 7.2 adjusted with $\mathrm{KOH}$; osmolarity, $280 \mathrm{mOsm} / \mathrm{L}$ ). Voltage was filtered at 10 $\mathrm{kHz}$ and digitized at $50 \mathrm{kHz}$ using an ITC-16 interface (InstruTech, Port Washington, NY). AxoGraph software (Molecular Devices, Foster City, CA) was used for both acquisition and analysis. Experiments were conducted at near physiological temperatures $\left(\sim 34^{\circ} \mathrm{C}\right)$ unless otherwise stated. Pooled data are presented as mean \pm SEM. Statistical tests used a Student's $t$ test or ANOVA at a level of significance of 0.05 .

Voltage imaging was performed at room temperature $\left(22-26^{\circ} \mathrm{C}\right)$, unless otherwise stated, using the voltage-sensitive dye JPW1114 (Invitrogen, San Diego, CA) or JPW3028 (synthesized and kindly provided by J. P. Wuskell and L. M. Loew, University of Connecticut, Farmington, CT). Recording pipettes were back-filled with intracellular solution containing 1-3 mg/ml dye, and 45-60 min was allowed for the passive transfer of dye into the neuron before the patch pipette was removed. The dye-filled neuron was left undisturbed for $>2 \mathrm{~h}$ before being repatched with a dye-free pipette. Orthodromic APs were evoked by extracellular synaptic stimulation close to the soma $(50-100 \mu \mathrm{m})$ within layer 5 using a standard patch pipette filled with extracellular solution $(22-59 \mu \mathrm{A} ; 100$ $\mu \mathrm{s})$ or via brief somatic current injection $(0.8-2.3 \mathrm{nA} ; 2 \mathrm{~ms})$. Antidromic APs were evoked by extracellular synaptic stimulation near the border of layer 6 and the white matter. Fluorescence changes during APs were detected with a back-illuminated cooled CCD camera (Red Shirt Imaging, Fairfield, CT) operating at $10 \mathrm{kHz}(80 \times 12$ pixel resolution $)$ using Neuroplex software (Red Shirt Imaging). Excitation was achieved using either a 150 W Xenon (Optiquip, Highland Mills, NY) or 100 W Halogen lamp (Olympus, Tokyo, Japan) gated by a shutter (Uniblitz Electronics, Rochester, NU) and appropriate filters (excitation, $520 \pm 45 \mathrm{~nm}$; dichroic, $570 \mathrm{~nm}$; emission, $>610 \mathrm{~nm}$ ). Illumination was limited to $60 \mathrm{~ms}$ per trial. To maximize the signal-to-noise ratio, we used regions of interest (ROIs) of three pixels length $(\sim 10 \mu \mathrm{m})$ and two pixel width $(\sim 7 \mu \mathrm{m})$ and typically averaged $100-150$ responses. Results obtained from averages of the first and last 30 trials gave similar results. Before averaging, individual trials (see Fig. $1 C$ ) were aligned using the AP recorded via the somatic recording pipette. This procedure removed trial-to-trial temporal jitter in the timing of AP generation. Fluorescence changes were not filtered, and the amplitude of the optical signal was expressed as the percentage change in light intensity divided by the resting light intensity $(\Delta F / F)$. The onset latency of fluorescent signals was measured at half amplitude after fitting a linear regression to the rising phase centered around the region of maximum slope (see Fig. $1 D$ ). Similar results were obtained when using onset latency based on the time to reach $10 \%$ of peak amplitude. Voltage-sensitive dye (VSD) experiments were discontinued if the amplitude of somatic APs decreased by $>5 \%$ during the recording procedure (average AP amplitude at the end of analysis $99.8 \pm$ $3.2 \mathrm{mV})$.

AP characteristics were analyzed during focal application of either a low-sodium extracellular solution $\left(125 \mathrm{~mm} \mathrm{C}_{5} \mathrm{H}_{14} \mathrm{NO} \cdot \mathrm{Cl}, 25 \mathrm{~mm}\right.$ glucose, $25 \mathrm{~mm} \mathrm{NaHCO}_{3}, 3 \mathrm{~mm} \mathrm{KCl}, 1.25 \mathrm{~mm} \mathrm{NaH}_{2} \mathrm{PO}_{4}, 2 \mathrm{~mm} \mathrm{CaCl}_{2}, 1 \mathrm{~mm}$ $\mathrm{MgCl}_{2}$, and $5 \mu \mathrm{M}$ Alexa Fluor 568, pH 7.4, osmolarity 300-310 mOsm/L) or extracellular solution containing tetrodotoxin (TTX; $10 \mu \mathrm{M}$ ). The pipette solution was pressure ejected (5-15 psi) from a standard patch pipette to visually identified regions of the axon using a Picospritzer II (General Valve, Fairfield, NJ). Neurons were loaded with a fluorescent dye (low-sodium solution, $10 \mu \mathrm{m}$ Alexa Fluor 568; TTX, $3 \mathrm{mg} / \mathrm{ml} \mathrm{JPW}$ 3028) via the somatic recording pipette and the axon as well as the location of the puffer pipette imaged with a confocal microscope (FV300, Olympus; or LSM 510, Zeiss, Thornwood, NY) or CCD camera (Red Shirt Imaging). AP amplitude, rise time (10-90\%), and half width (full width at half-height) were measured from threshold, defined as the membrane potential at which the voltage rate of rise was $50 \mathrm{~V} / \mathrm{s}$. Lowsodium experiments were performed at near physiological temperatures $\left(\sim 34^{\circ} \mathrm{C}\right)$, and TTX experiments were performed at room temperature $\left(\sim 22^{\circ} \mathrm{C}\right)$.

In experiments in which neurons had axons of varying length (21-968 $\mu \mathrm{m}$ ), neurons were selected at random, and the length of axon was determined at the end of the experiments after loading with a fluorescent dye (10 $\mu \mathrm{m}$ Alexa Fluor 568) via the somatic recording pipette using a confocal microscope (FV300, Olympus; or LSM 510, Zeiss). Heterogeneity of axon length was a consequence of the brain slicing procedure. AP characteristics (threshold, amplitude, rise time) were determined from the first AP generated $>50 \mathrm{~ms}$ after the onset of a 1-s-long somatic current injection $(150-600 \mathrm{pA})$.

Oligodendrocytes were identified by their characteristic spherical cell body using DIC imaging and filled with the red fluorescent dye Alexa Fluor $568(10 \mu \mathrm{M})$. Myelinating processes were visualized using confocal microscope (LSM 510; Zeiss), and layer 5 pyramidal neurons with cell bodies immediately above (closer to the pia) myelinating processes of oligodendrocytes were selected and patched with patch pipettes containing the green fluorescent dye Oregon Green 488 (150 $\mu$ M; Invitrogen).

Extracellular recordings were made using standard patch pipettes (tip diameter, 2-3 $\mu \mathrm{m}$ ) filled with extracellular solution plus a fluorescent dye (10 $\mu \mathrm{M}$ Alexa Fluor 568; Invitrogen). The axon was visualized after filling neurons via the somatic whole-cell recording pipette, which was also filled with fluorescent dye (10 $\mu \mathrm{M}$ Alexa Fluor 568). The axon, as well as the extracellular recording pipette, were imaged using a confocal microscope (LSM 510; Zeiss) enabling the extracellular recording pipette to be positioned under visual control in close apposition to the initial segment and site of the first axon collateral. APs were evoked by somatic current injection, and the onset latency of the extracellular signal relative to the somatic AP was determined from a linear regression fitted to the region of maximum slope, as for VSD responses.

In experiments testing the effect of GABA application to the initial segment, an extracellular solution containing GABA (100 $\mu \mathrm{M})$ was pressure ejected ( $5 \mathrm{~ms} ; 12 \mathrm{psi}$ ) from a standard patch pipette using a Picospritzer II (General Valve). APs were evoked by brief somatic current injection (2-5 nA; $2 \mathrm{~ms}$ ) in the absence (control) or just after (20-50 ms) GABA application, alternating between control and GABA in blocks of $\sim 30$ trails.

\section{Results}

\section{Determination of the site of AP initiation with VSDs}

We first determined the temporal resolution of the camera-based system used to detect signals with VSDs. To test this, we generated AP-like light responses by driving a light-emitting diode (LED) with an AP voltage command within its linear range. We then shifted the onset of the AP command in $20 \mu$ s steps and recorded the LED output using a photodiode sampling at 100 $\mathrm{kHz}$ or our camera-based system sampling at $10 \mathrm{kHz}$ (Fig. $1 \mathrm{~A}$ ). Despite a 10-fold lower temporal resolution, the camera-based detection system could reliably resolve changes in AP-light response onset with a resolution of $<20 \mu \mathrm{s}$ (Fig. $1 \mathrm{~A}$, bottom). The ability to detect time differences less than the sample interval comes about, because the AP rise time is slow enough that there are multiple sample points on the rising phase. Any detection system that can describe the rising phase of an event can resolve time differences significantly less than the sample interval.

Subsequently, we made whole-cell recordings from the soma of cortical layer 5 pyramidal neurons with pipettes back-filled 
with the voltage-sensitive dye JPW3028 or JPW1114 (1-3 mg/ml). After an initial period of filling ( $\sim 60 \mathrm{~min})$, the pipette was removed and the dye was allowed to distribute within the neuron for at least $2 \mathrm{~h}$ before repatching using a pipette solution without dye (Antic, 2003). Intracellular filling with VSDs led to a small but significant reduction in AP amplitude by, on average, $4 \%$ (control, $109.3 \pm 1.4$ vs $105.3 \pm$ $2.0 \mathrm{mV}$ after repatching). There was no statistically significant effect on AP rise time (control, $0.24 \pm 0.01 \mathrm{~ms}$ vs $0.24 \pm$ $0.01 \mathrm{~ms}$ after repatching), threshold (control, $13.4 \pm 1.1 \mathrm{mV}$ depolarized to rest vs $15.5 \pm 1.8 \mathrm{mV}$ after repatching), or halfwidth (control, $1.2 \pm 0.1 \mathrm{~ms}$ vs $1.3 \pm 0.1$ ms after repatching). The axon was identified as a nonspiny process that originated from the base of the soma and projected toward the white matter (Fig. 1B).

Single APs were elicited via somatic current injection or extracellular synaptic stimulation within layer 5 , and simultaneous fluorescence measurements were made at the soma and different axonal regions at high temporal resolution $(10 \mathrm{kHz})$ (Fig. $1 B$ ). Comparison of the recorded somatic voltage with the fluorescence change at the soma indicated that the waveform of the somatic AP could be reliably captured using VSDs (Fig. 1C). Simultaneously sampling the fluorescent change during APs in the axon, soma, and proximal apical dendrite showed that, consistent with previous findings (Stuart and Häusser, 1994; Stuart et al., 1997a), APs are initiated in the axon of layer 5 pyramidal neurons (Fig. 1D).

To identify the site of AP initiation in the axon, we simultaneously recorded the VSD signal at multiple locations in the axon during AP generation (Fig. 2A). The spatial resolution of these measurements was $\sim 10 \mu \mathrm{m}$. We then compared the onset latency of axonal fluorescence changes during APs with the fluorescence change recorded at the soma (Fig. $2 B$ ). By definition, the site in the axon with the shortest onset latency is the site of AP initiation. On average, the shortest onset latency was $35.6 \pm 2.3$ $\mu \mathrm{m}$ from the axon hillock (Fig. $2 C)(n=34)$. Similar results were obtained in experiments in which APs were evoked by synaptic stimulation $(35.0 \pm 3.8 \mu \mathrm{m} ; n=7)$, brief $(2 \mathrm{~ms} ; 35.7 \pm 2.7 \mu \mathrm{m}$; $n=27)$ or steady-state somatic current injection $(32.1 \pm 4.2 \mu \mathrm{m}$; $n=7$ ), as well as during AP initiation at close to physiological temperatures $\left(\sim 34^{\circ} \mathrm{C} ; 35.0 \pm 5.8 \mu \mathrm{m} ; n=3\right)$. Together, these data indicate that APs in layer 5 pyramidal neurons are initiated in a localized region of the axon $\sim 35 \mu \mathrm{m}$ from the axon hillock.

Once initiated, APs propagated down the axon in a saltatory manner, as depicted by reoccurring shortenings in onset latency (Fig. $2 B$ ). These shortenings in onset latency were aligned with axonal branch points in a subset of neurons $(n=6)$ where AP propagation was tracked over $\geq 200 \mu \mathrm{m}$ of the axon (Fig. $2 B$, gray arrows). Previous work indicates axonal branch points commonly arise at nodes of Ranvier (Sloper and Powell, 1979; Fraher and Kaar, 1984).

These results contrast with that observed when APs were evoked by antidromic stimulation via extracellular stimulation near the border of layer 6 and the white matter (Fig. 2C) $(n=6)$. During antidromic APs, the site in the axon with the shortest onset latency was $>200 \mu \mathrm{m}$ from the soma (Fig. $2 C$ ), consistent with a site of AP initiation in the distal axon (close to the stimulation pipette). These findings indicate that our VSD recordings are sensitive enough to detect differences in the site of AP initiation during orthodromic and antidromic stimulation.

\section{Mapping the site of AP initiation using low-sodium applications}

Because VSDs have a low signal-to-noise ratio and potential problems because of toxicity (although this was minimal in our experiments), we used alternative methods to support our findings using VSDs. To test the role of different axon regions in AP initiation, we investigated the impact of locally reducing inward current flow through voltage-activated sodium channels via brief applications of a low-sodium extracellular solution. Initial experiments used a low-sodium extracellular solution, because this should allow a rapid and local reduction in sodium current with immediate recovery. The rationale was that if AP initiation occurs in the proximal region of the axon, as the VSD experiments suggest, then reducing sodium current in this region would be expected to significantly influence AP properties. Focal application of low-sodium extracellular solution to the proximal axon led to a significant increase in AP threshold, as well as a decrease in the amplitude of the somatic AP (Fig. 3A). These effects were greatest 


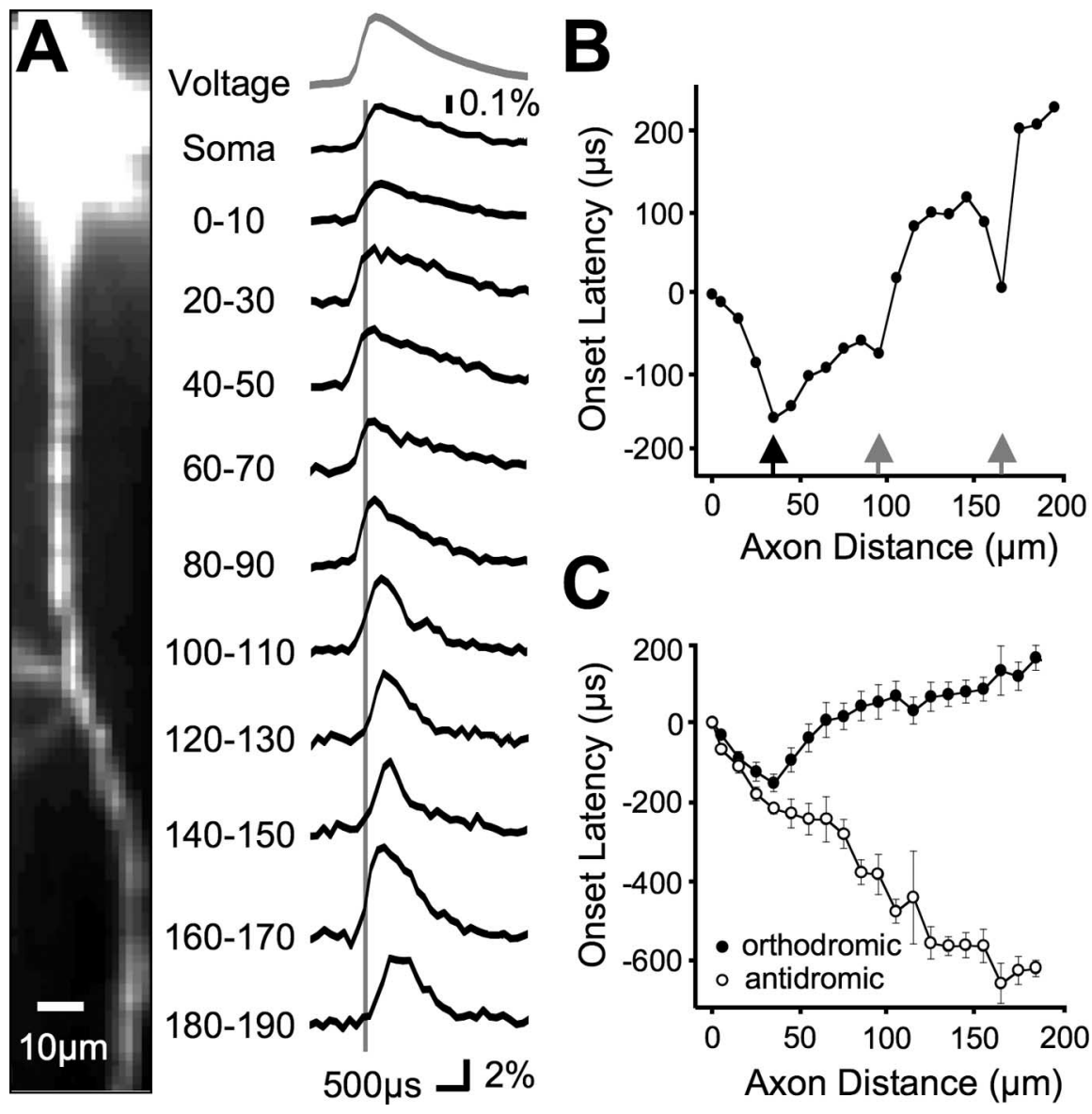

Figure 2. Imaging AP initiation with VSDs. A, Left, High-magnification image of the axon of a layer 5 pyramidal neuron filled with VSD. Right, Average fluorescence change $(\Delta F / F)$ of 130 individually aligned APs recorded at the indicated axonal locations. APs were evoked by somatic current injection. $\boldsymbol{B}$, Onset latency of axonal VSD signals relative to the somatic response $(0 \mu \mathrm{m})$ plotted against distance from the axon hillock for the data in $\boldsymbol{A}$. The site of AP initiation is indicated by a black arrow. Gray arrows indicate location of axonal branch points. $C$, Average onset latency of axonal VSD signals relative to the somatic response plotted against distance from the axon hillock for orthodromic (filled circles; $n=34$ ) and antidromic APs (open circles; $n=6$ ). Data for orthodromic APs were pooled from experiments in which APs were evoked by brief somatic current injection $(n=27)$ and synaptic stimulation $(n=7)$.

during applications $20 \mu \mathrm{m}$ from the hillock and decreased back to control levels at axonal sites $>60 \mu \mathrm{m}$ from the soma (Fig. $3 B, C)$. On average, low-sodium applications $20 \mu \mathrm{m}$ from the hillock increased AP threshold by $4.5 \pm 1.0 \mathrm{mV}$ and decreased AP amplitude by $8.8 \pm 1.5 \mathrm{mV}(n=8 ; p<0.05)$. Low-sodium applications also slightly increased AP rise time, with the greatest effect during applications to the axon hillock (53 $\pm 9 \mu \mathrm{s} ; n=8$; $p<0.05)$. Focal application of the sodium channel antagonist TTX (10 $\mu \mathrm{M} ; 5 \mathrm{~ms})$ to the proximal axon ( $\sim 20 \mu \mathrm{m}$ from the axon hillock) also led to a significant increase in AP threshold (by $14.6 \pm 4.5 \mathrm{mV}$ ) and rise time (by $137 \pm 45 \mu \mathrm{s}$ ) and a decrease (by $28.2 \pm 6.0 \mathrm{mV})$ in AP amplitude $(n=6 ; p<0.05)$ (Fig. $3 D)$. These findings indicate that locally reducing current flow through voltage-activated sodium channels has the greatest impact on AP properties when applied to proximal axonal sites and are consistent with the results obtained using VSDs that the site of $\mathrm{AP}$ initiation resides in the proximal region of the axon.

\section{Influence of axon length on AP properties}

If the region of axon responsible for AP initiation was severed during the slicing procedure, one would expect AP properties such as threshold and amplitude to be altered. The minimum length of axon required to generate APs with "normal" properties should therefore give an indication of the site in the axon where APs are initiated. To investigate this issue, we recorded from layer 5 pyramidal neurons with axons randomly severed at lengths ranging from 21 to $968 \mu \mathrm{m}(n=108)$ during the brain slicing procedure. As with the low-sodium experiments, these experiments were performed at near physiological temperatures $\left(\sim 34^{\circ} \mathrm{C}\right)$. The length of axon was determined using either confocal microscopy after filling with a fluorescent dye (Alexa 568) (Fig. 4A) or light microscopy after filling with biocytin $(3 \mathrm{mg} / \mathrm{ml})$ via the somatic pipette solution. The temporal derivative of membrane voltage $(d V / d t)$ can be used to dissociate the individual components that make up the somatic AP (Coombs et al., 1957). This has led to the conclusion that somatic APs exhibit two distinct components, which result from the sequential activation of the axon initial segment and somatodendritic compartment (Coombs et al., 1957). We observed two distinct components of the temporal derivative of the somatic AP in all layer 5 pyramidal neurons regardless of the axonal length (Fig. $4 B$ ). This finding suggests that the generation of APs even in neurons with very short axons follows the sequence of activation of the initial segment followed by the somatodendritic membrane.

Both the voltage change required to reach somatic AP threshold and AP amplitude were similar in neurons with axonal lengths ranging from 25 to nearly $1000 \mu \mathrm{m}$ (Fig. 4C). Furthermore, there was no statistically significant correlation between axonal length and AP rise time $(92.6 \pm 2$ $\mu \mathrm{s} ; p=0.08)$, half-width $(395 \pm 9 \mu \mathrm{s} ; p=$ $0.58)$, or the resting membrane potential $(-66 \pm 0.48 \mathrm{mV} ; p=$ 0.39 ; data not shown). These observations indicate that neurons with very short axons $(\sim 30 \mu \mathrm{m})$ contain the appropriate machinery to produce normal APs, and that more distal regions of the axon are not required for AP generation.

\section{Anatomical identification of the site of AP initiation}

To determine how the site of AP initiation in the proximal axon site relates to axon myelination, we made sequential whole-cell recordings from oligodendrocytes and layer 5 pyramidal neurons using patch pipettes filled with different fluorescent dyes (Alexa 568 and Oregon Green 488) and imaged oligodendrocyte and axonal processes using confocal microscopy. The aim of these experiments was to label oligodendrocytes supplying the first region of myelin to layer 5 pyramidal neuron axons, thereby defining the length of the initial segment as well as the location of the first node of Ranvier, which by definition will be at the end of the first myelinated segment. Oligodendrocytes were identified anatomically as having spherical cell bodies with an average diameter of $7.8 \pm 0.6 \mu \mathrm{m}$ located in layers 5 and 6 and were electrically in-excitable. Each oligodendrocyte had multiple myelin processes of differing lengths and widths that ensheathed the axons of several neurons. On six occasions, we were able to fill a layer 5 

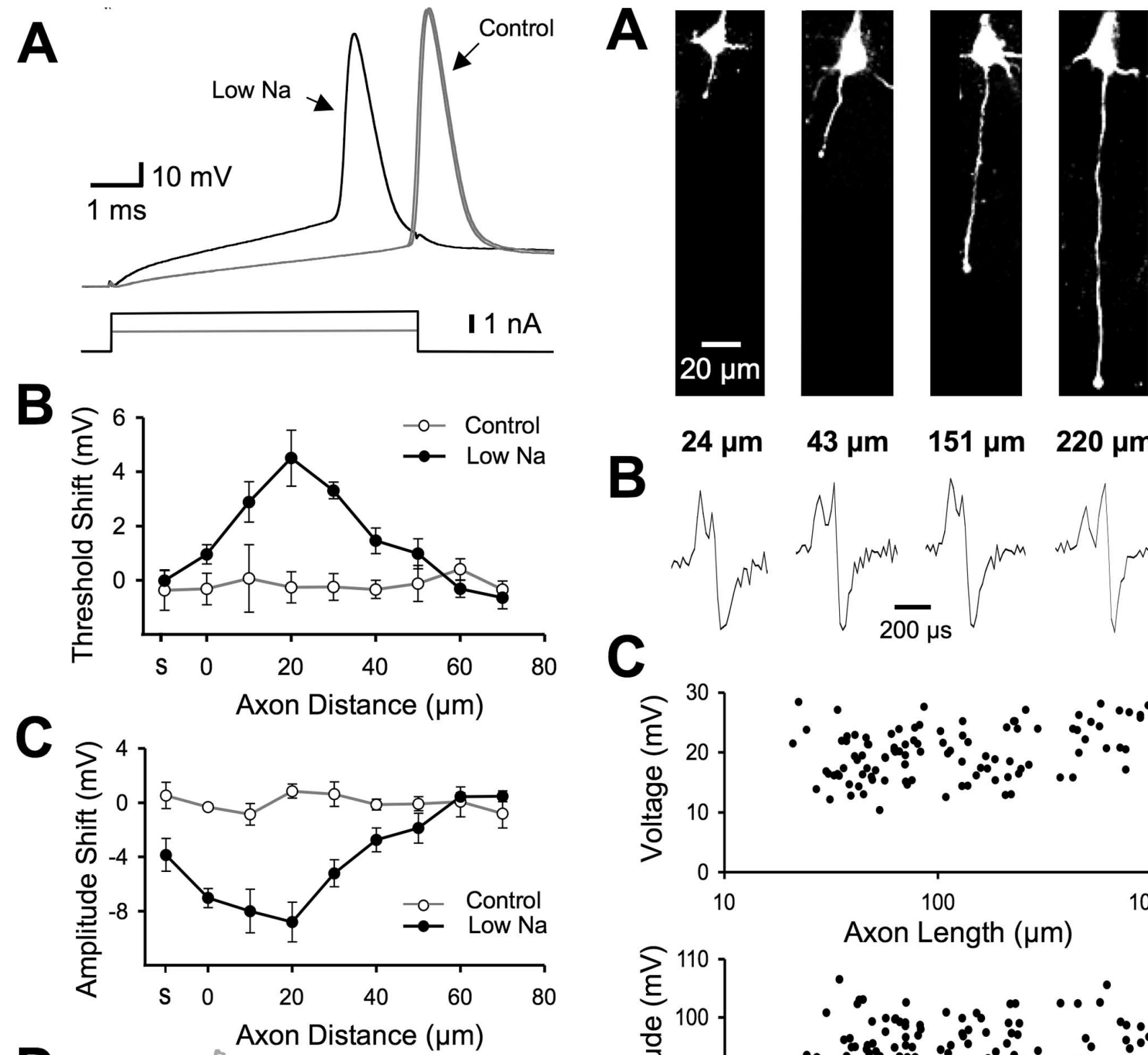

$D$
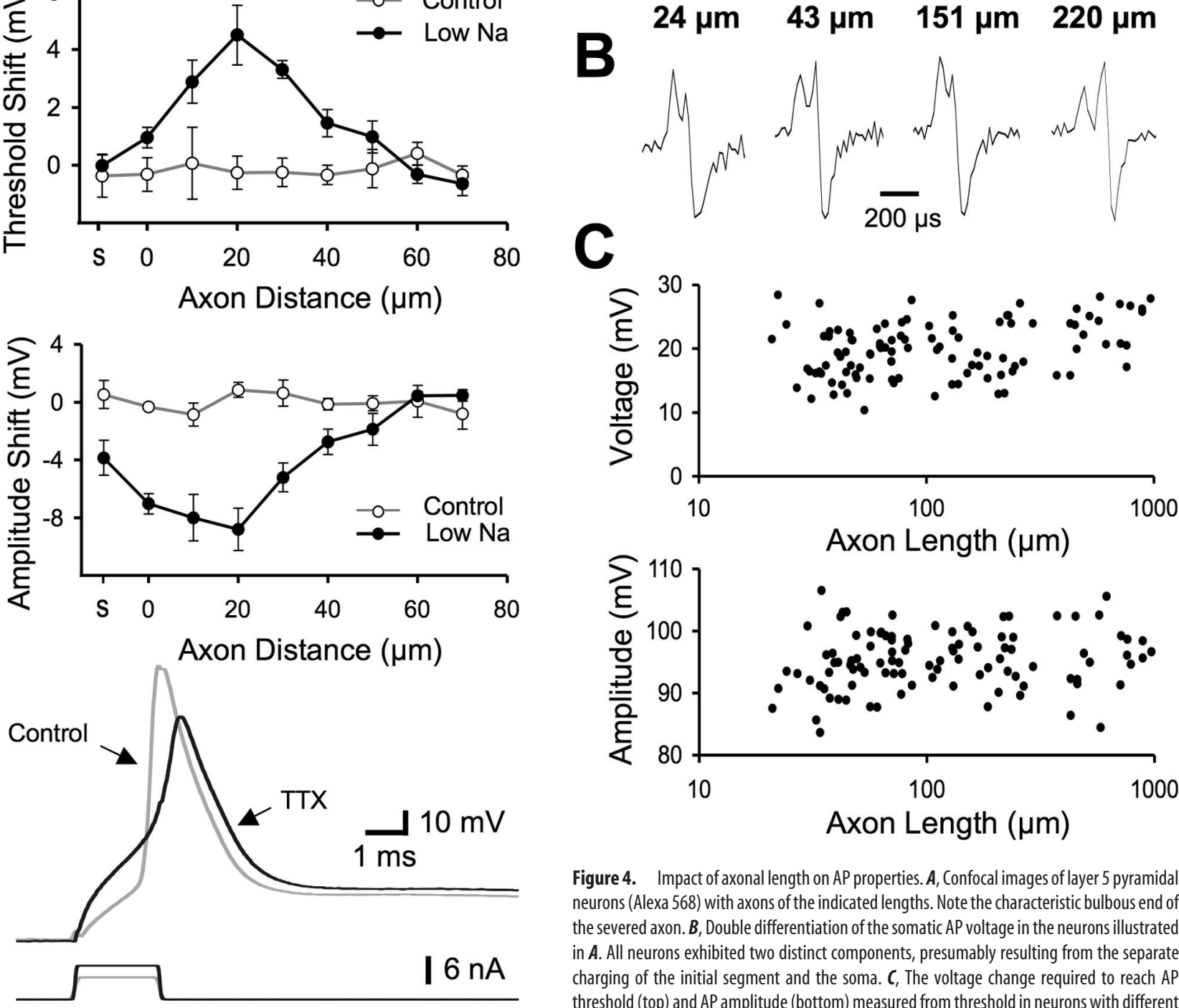

Figure 4. Impact of axonal length on AP properties. $A$, Confocal images of layer 5 pyramidal neurons (Alexa 568) with axons of the indicated lengths. Note the characteristic bulbous end of the severed axon. $\boldsymbol{B}$, Double differentiation of the somatic AP voltage in the neurons illustrated in $\boldsymbol{A}$. All neurons exhibited two distinct components, presumably resulting from the separate charging of the initial segment and the soma. $C$, The voltage change required to reach AP threshold (top) and AP amplitude (bottom) measured from threshold in neurons with different

Figure 3. Impact of local reductions in sodium current on AP properties. A, Example of APs evoked by somatic current injection during brief $(20 \mathrm{~ms})$ low-sodium application to the axon initial segment (20 $\mu \mathrm{m}$ from the axon hillock) compared with control APs recorded before and after low-sodium application. Low-sodium extracellular solution led to a significant increase in both AP threshold and the amplitude of the somatic current step (bottom) required to reach threshold. $\boldsymbol{B}$, Average shift in somatic AP threshold and amplitude ( $($ ) relative to control during brief application of low-sodium solution (filled circles) or normal extracellular solution (open circles) to the axon at different locations. $\mathrm{S}$, Soma. The axon hillock is $0 \mu \mathrm{m}$. D, APs evoked by somatic current injection during brief $(5-10 \mathrm{~ms})$ focal application of TTX (10 $\mu \mathrm{m})$ to the axon initial segment ( $20 \mu \mathrm{m}$ from the axon hillock) compared with a control AP recorded before TTX application.

pyramidal neuron receiving myelination from one of these oligodendrocyte processes (Fig. 5A). The average distance of the myelin to the axon hillock was $39.9 \pm 5.2 \mu \mathrm{m}$, and the average length of this myelin process was $50.2 \pm 9.6 \mu \mathrm{m}(n=6)$. These results indicate that the length of the unmyelinated initial segment region is $\sim 40 \mu \mathrm{m}$, the length of the first region of myelination is 

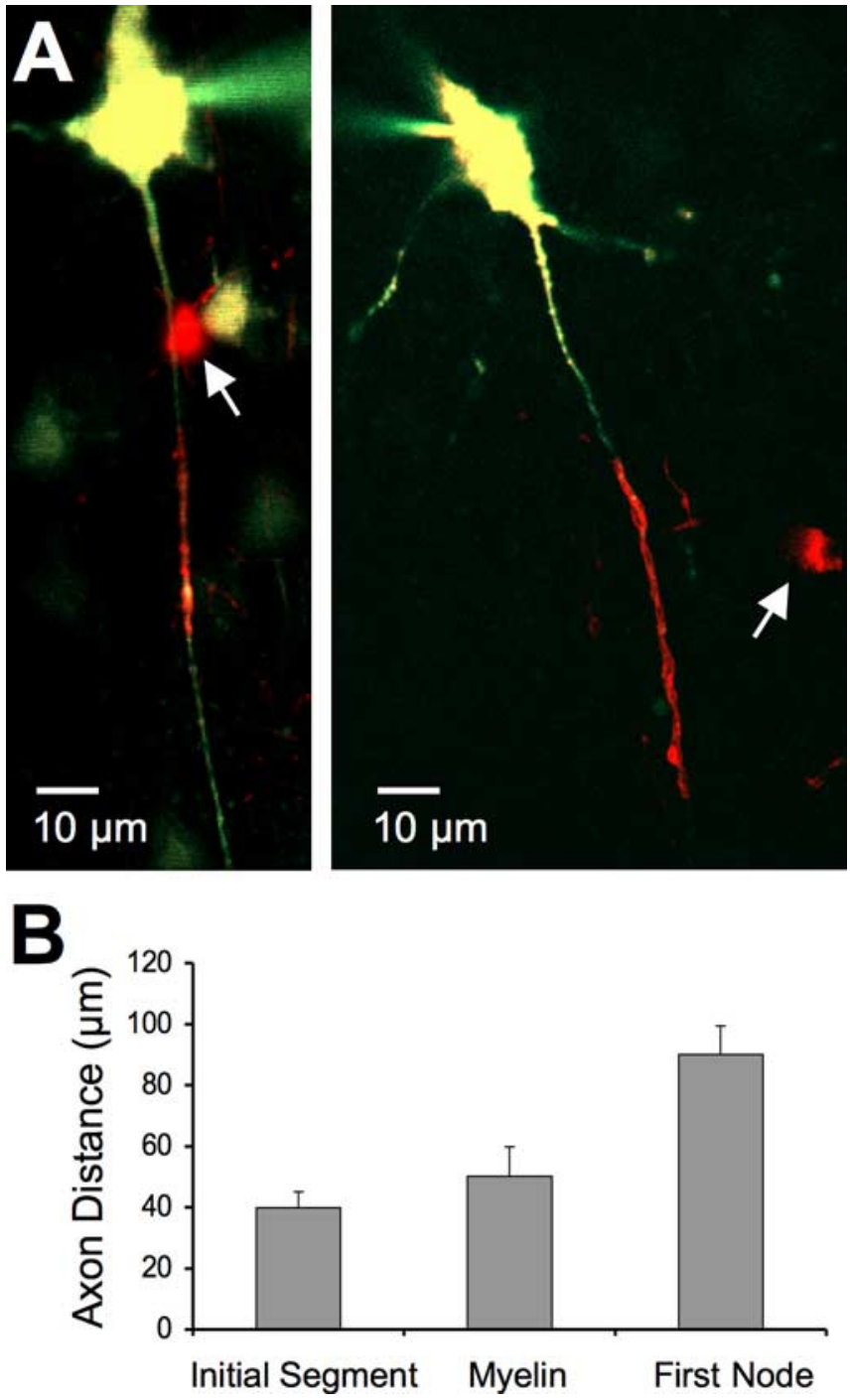

Figure 5. Morphological identification of the axon initial segment. $\boldsymbol{A}$, Two examples of confocal microscope images of oligodendrocytes and associated processes (red; Alexa Fluor 568) myelinating the initial region of the axon of a layer 5 pyramidal neuron (yellow; Oregon Green 488). In both examples, the oligodendrocyte cell body can be identified as a red sphere (arrow). B, Pooled data $(n=6)$ of the average distance from the axon hillock to the start of the first myelin process $(\sim 40 \mu \mathrm{m})$, the length of the first myelin process $(\sim 50 \mu \mathrm{m})$, and the distance from the axon hillock to the end of the first myelin process $(\sim 90 \mu \mathrm{m})$, indicating the distance to the first node of Ranvier. Error bars represent SEM.

$\sim 50 \mu \mathrm{m}$, and therefore the first node of Ranvier occurs $\sim 90 \mu \mathrm{m}$ from the soma (Fig. 5B).

It is very likely that these oligodendrocyte processes are indeed the first regions of myelination of layer 5 pyramidal neuron axons, because oligodendrocyte processes shorter than $40 \mu \mathrm{m}$ were never observed. In addition, the oligodendrocyte process that ensheathed the proximal region of the layer 5 pyramidal neuron axon had a larger diameter $(1.5 \pm 0.2 \mu \mathrm{m})$ than other oligodendrocyte processes $(1.0 \pm 0.1 \mu \mathrm{m})$. In fact, we specifically targeted layer 5 pyramidal neurons located superficial to the largest diameter oligodendrocyte process. Combining these anatomical data on the length of the axon initial segment $(40 \mu \mathrm{m})$ with our observed site of AP initiation $\sim 35 \mu \mathrm{m}$ from the axon hillock indicates that the site of AP generation in layer 5 pyramidal neurons is in the distal region of the axon initial segment.

\section{Role of the first node of Ranvier}

The original studies on the origin of AP initiation suggested that initiation may occur at the first node of Ranvier (Coombs et al. 1957), with more recent studies supporting this idea (Gogan et al., 1983; Colbert and Johnston, 1996; Clark et al., 2005). The anatomical data presented above suggests that this site will be $\sim 90 \mu \mathrm{m}$ from the axon hillock. Because it is known that branch points commonly arise from nodes of Ranvier (Sloper and Powell, 1979; Fraher and Kaar, 1984), we searched for the first axonal collateral process of layer 5 pyramidal neuron axons after filling neurons with a fluorescent dye (Alexa 568) using confocal microscopy (Fig. $6 A$ ). On average, this collateral process was $105 \pm$ $8 \mu \mathrm{m}(n=6)$ from the axon hillock, consistent with the idea that it represented the first node of Ranvier. After identification of the putative first node of Ranvier, we investigated its potential role in AP initiation using extracellular recordings, brief applications of low-sodium extracellular solution and TTX (as in Fig. 3), and VSDs (as in Fig. 2). APs were evoked by somatic current injection, and the onset of the extracellularly recorded voltage response at the first axon collateral was compared with that recorded in the axon initial segment. In all cases, the onset of extracellularly recorded response at the first axon collateral occurred significantly later than the response recorded in the axon initial segment of the same neuron (Fig. $6 B)(n=7 ; 40 \mu \mathrm{m},-144 \pm 13 \mu$ s vs $-78 \pm 17$ $\mu$ s relative to the somatic AP; $p<0.05)$. The role of the first node of Ranvier in AP initiation was also investigated with focal applications of low-sodium extracellular solution to the first axon branch point $(n=5)$. Somatic AP threshold was unaffected by low-sodium applications to the first branch point $(-1.7 \pm 0.2$ $\mathrm{mV} ; n=5 ; p=0.53$ ), whereas similar applications to the initial segment of the same neurons caused a statistically significant shift in AP threshold (Fig. 6C) $(+5.1 \pm 0.8 \mathrm{mV} ; n=5 ; p<0.05)$. Consistent with this result, focal applications of TTX $(10 \mu \mathrm{M})$ targeted to the first branch point did not affect somatic AP threshold $(+0.8 \pm 1 \mathrm{mV} ; n=6 ; p=0.94)$. Finally, we investigated the role of the putative first node in AP initiation using VSDs (Fig. 6D). Relative to the somatic response, the onset latency of the AP fluorescence change at the first collateral branch point was delayed by on average $+20 \pm 20 \mu$ s $(n=13)$. In contrast, the onset latency of the VSD signal at the distal axon initial segment (35 $\mu \mathrm{m}$ from the soma) preceded the somatic response by on average $-150 \pm 10 \mu \mathrm{s}(n=34)$. Together, these data indicate that the first node of Ranvier is not the site of AP initiation in layer 5 pyramidal neurons.

\section{Impact of axonal inhibition}

The axon initial segment of layer 5 pyramidal neurons receives GABAergic inputs from chandelier cells (Peters et al., 1982; Freund et al., 1983). To test whether activation of these inhibitory inputs alters the site of AP initiation, we locally applied GABA $(100 \mu \mathrm{M})$ to the axon initial segment. These GABA applications inhibited AP firing, leading to a significant increase in the amount of somatic current required to initiate APs (Fig. 7A) (control, $1.24 \pm 0.01 \mathrm{nA}$ vs GABA, $2.75 \pm 0.05 \mathrm{nA} ; p<0.05$ ), however, had no significant effect on the site of AP initiation (Fig. $7 B, C)(n=9)$. These data illustrate that the site of AP initiation is robust even under conditions of axonal inhibition.

\section{Development of action potential initiation and propagation}

Early investigations of AP propagation in myelinated axons indicated propagation occurs in a "saltatory" manner, progressing along the axon by jumping between active nodes of Ranvier (Huxley and Stampfli, 1949; Bishop and Levick, 1956). As indi- 


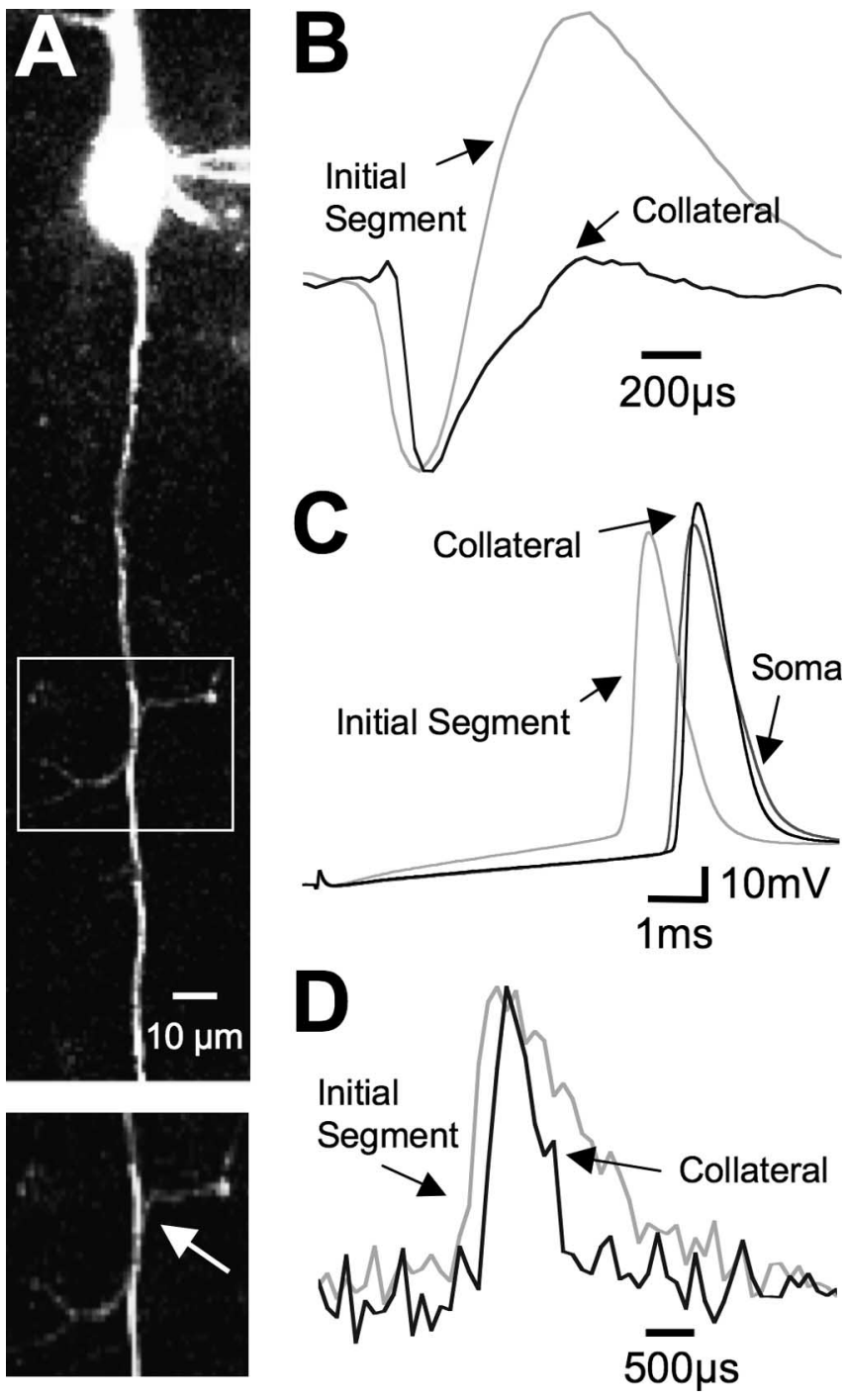

Figure 6. Role of the first node of Ranvier in AP initiation. $A$, Top, Confocal image of a layer 5 pyramidal neuron axon (Alexa Fluor 568) with two collateral processes. Bottom, Magnification of the boxed region showing the first branch point (arrow). The first collateral process was 100 $\mu \mathrm{m}$ from the axon hillock and presumably represents the first node of Ranvier. $\boldsymbol{B}$, Normalized extracellular voltage responses during APs evoked by somatic current injection and recorded at the initial segment $40 \mu \mathrm{m}$ from the axon hillock (gray; average of 110 trails) and at the first axon branch point (black; $127 \mu \mathrm{m}$ from the axon hillock; average of 110 trails). The onset of the extracellular voltage response recorded at the initial segment precedes that recorded at the first branch point, suggesting AP initiation does not occur at the first node of Ranvier. C, APs evoked by somatic current injection during application of low-sodium solution to the initial segment, soma, and first axon collateral. Focal application of low-sodium extracellular solution only significantly influenced AP threshold when applied to the axon initial segment. $\boldsymbol{D}$, Average fluorescence change $(\triangle F / F)$ of 140 individually aligned somatically evoked APs recorded at the initial segment ( $35 \mu \mathrm{m}$ from the hillock) and at the first collateral branch point ( $95 \mu \mathrm{m}$ from the hillock). The fluorescence change at the initial segment occurs before the fluorescence change recorded at the first collateral, illustrating that the AP occurs at the initial segment before it proceeds to the first node of Ranvier.

cated in Figure 2, a form of saltatory conduction was observed during orthodromic conduction of APs away from the site of AP initiation. This was represented by reoccurring shortenings in onset latency of VSD signals at distant axon locations and was observed during AP generation by synaptic stimulation $(n=6)$ and somatic current injection (Fig. $8 A, C)(n=8)$. In many cases, axonal sites of shortest onset latency were clearly associated with axonal branch points (Fig. $2 \mathrm{~B}$ ). Similar reoccurring shortenings
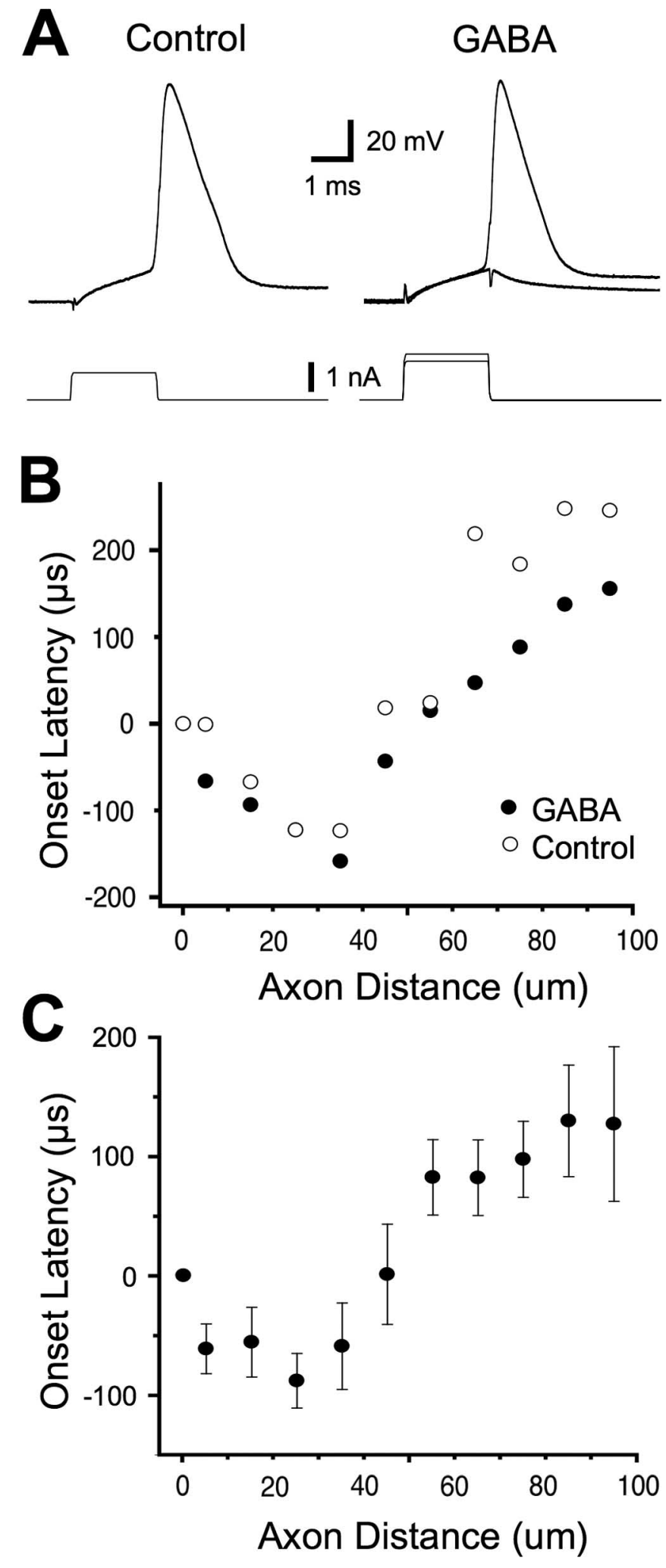

Figure 7. The effect of inhibition on AP initiation. $A$, Left, AP evoked by somatic current injection during control (no GABA). Right, Focal application of GABA (100 $\mu \mathrm{M})$ to the initial segment inhibited AP generation, which could be re-established by increasing the amplitude of the somatic current pulse. $B$, Onset latency of axonal VSD signals relative to the somatic response $(0 \mu \mathrm{m})$ plotted against distance from the axon hillock during GABA application to the initial segment (filled circles) and control (open circles). $C$, Average onset latency of axonal VSD signals relative to that at the soma plotted against distance from the axon hillock during GABA application to the initial segment $(n=9)$. 

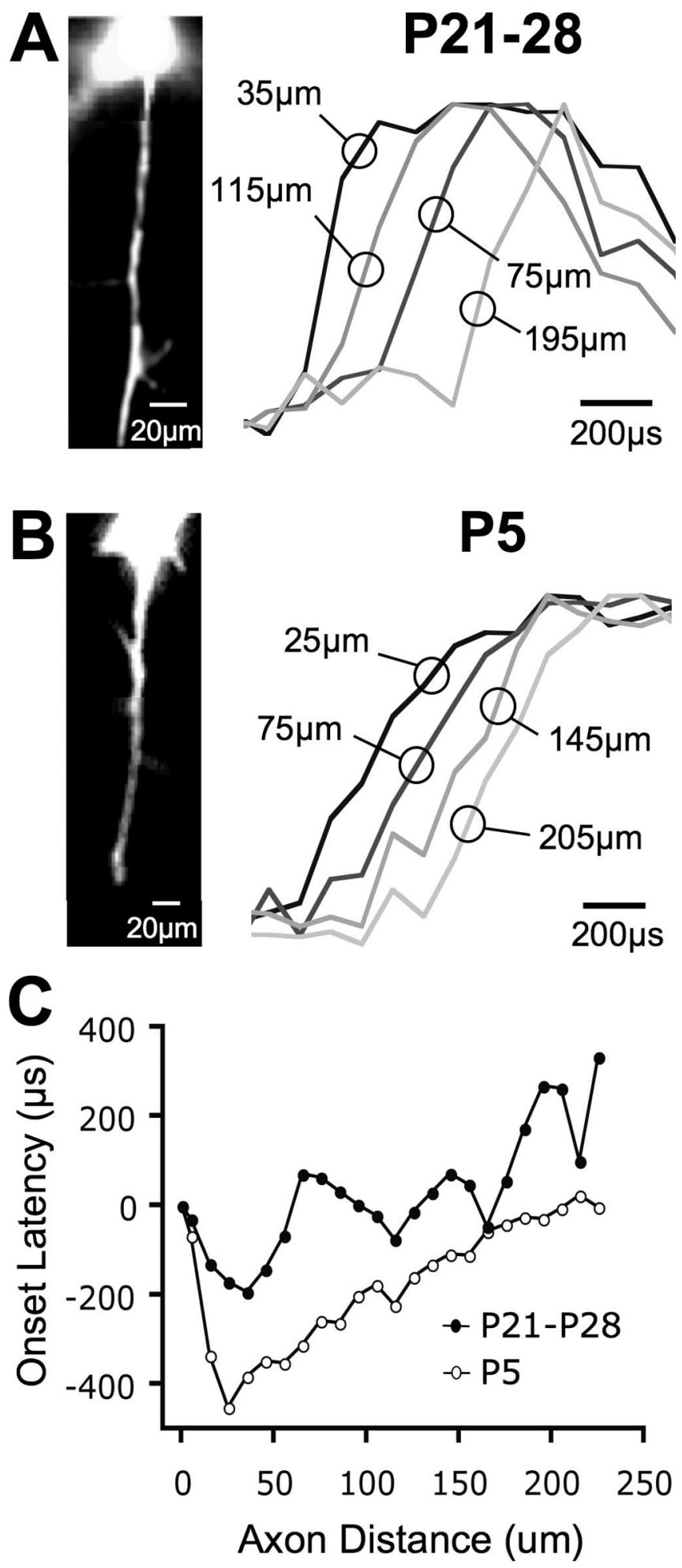

Figure 8. Development of AP initiation and propagation. $A$, Left, Fluorescent image of a mature (P21-P28) layer 5 pyramidal neuron filled with VSD. Right, Average fluorescence signal $(\Delta F / F)$ measured at the indicated locations along the axon (average, 100 trials). The fluorescence signal at the first branch point, $\sim 115 \mu \mathrm{m}$ from the hillock, occurs before the fluorescence signal recorded in the preceding axon region (75 $\mu \mathrm{m}$ from the hillock). $\boldsymbol{B}$, Left, Fluorescent image of an immature (P5) layer 5 pyramidal neuron filled with VSD. Right, Average fluorescence signal $(\Delta F / F)$ measured at the indicated locations along the axon (average, 140 trials). Note the sequential propagation of the fluorescence signal along the axon. C, Onset latency of axonal VSD signals relative to the somatic response $(0 \mu \mathrm{m})$ plotted against distance from the axon hillock for the immature (P5) and mature (P21-P28) layer 5 pyramidal neurons shown in $A$ and $\boldsymbol{B}$. APs were evoked by brief somatic current injection. in AP onset latency were observed in preparations from 2-weekold rats, which lack myelination ( $n=5$; data not shown), but were absent in immature preparations from 5-d-old [postnatal day 5 (P5)] rats (Fig. $8 B, C)(n=8)$. Layer 5 pyramidal neuron axons in $\mathrm{P} 5$ rats were typically $<250 \mu \mathrm{m}$ long ending in a growth cone (Fig. $8 B$ ). Despite their immature state, AP initiation in P5 neurons occurred at a similar distance from the axon hillock as in mature preparations (Fig. 8C). These developmental differences in $\mathrm{AP}$ conduction will require additional investigation and are likely to reflect differences in axonal sodium channel expression and localization before myelination as has recently been shown in retinal ganglion cell axons (Boiko et al., 2001).

\section{Discussion}

The discovery of electrical conduction in nerves by Galvani in the 18th century beckoned the question where within nerve cells electrical impulses, or APs, are generated. This question is fundamental to an understanding of how neurons integrate synaptic inputs. In this study, we provide direct evidence that APs in layer 5 pyramidal neurons are initiated $\sim 35 \mu \mathrm{m}$ from the axon hillock, at the distal axon initial segment. This was observed during AP generation under a variety of conditions, after axonal inhibition, and at different stages of development.

Using VSDs to assess the site of AP initiation has advantages and disadvantages. The main advantage is that it allowed us to image voltage simultaneously at multiple locations in the axon with high temporal $(10 \mathrm{kHz})$ and spatial $(\sim 10 \times 7 \mu \mathrm{m} ; 3 \times 2$ pixels) resolution. Disadvantages included a low signal-to-noise ratio (hence the need to average multiple trials) and the fact that VSDs can be toxic, although not very apparent in our experiments. For these reasons, we felt it necessary to support the results obtained with VSDs using alternative methods. First, we investigated the impact of reducing current flow through axonal voltage-activated sodium channels using brief $(20 \mathrm{~ms})$ applications of either a low-sodium external solution or TTX (Fig. 3). The reasoning here was that if the axon initial segment is responsible for AP initiation, then one would expect that reducing current flow through sodium channels at this location should lead to significant changes in AP threshold. Indeed, this is what we observed, with the greatest change in AP threshold during lowsodium and TTX applications occurring in the proximal axon. Presumably, the greatest effect of low-sodium applications was observed at $20 \mu \mathrm{m}$, rather than at the site of AP initiation $\sim 35$ $\mu \mathrm{m}$ from the axon hillock, because the maximum effect on AP threshold occurs when current flow over the entire axon initial segment is compromised. Given that the axon initial segment is $40 \mu \mathrm{m}$ long, it is not surprising that low-sodium applications at its midpoint $(20 \mu \mathrm{m})$ have the greatest effect. Although these experiments cannot be used to accurately pinpoint the site of AP initiation, they provide strong support for the notion that AP initiation occurs in the axon initial segment. Additionally, we investigated the impact of axonal length on AP properties (Fig. 4). As with the low-sodium and TTX experiments, these data indicated that the machinery necessary for AP initiation is contained within the axon initial segment, adding additional weight to the conclusions from the VSD experiments.

\section{Comparisons with previous studies}

A recent study in cerebellar Purkinje neurons, however, provided evidence that AP initiation can occur at the first node of Ranvier (Clark et al., 2005). In contrast, based on intracellular filling of the oligodendrocyte that provided the first myelinated segment to the axon of layer 5 pyramidal neurons, we conclude that the 
anatomical location of the site of AP initiation is the distal region of the axon initial segment. In addition, we directly addressed the possibility that APs are initiated at the first node of Ranvier using extracellular recording, and low-sodium and TTX applications are initiated at the first axon branch point (Fig. 6), which has been identified previously to often occur at the first node of Ranvier (Sloper and Powell, 1979). Consistent with the idea that this site represents the first node of Ranvier, the average distance from the axon hillock to the first branch point $(105 \mu \mathrm{m})$ was similar to the average distance from the axon hillock to the end of the first myelinated axon process $(90 \mu \mathrm{m})$. These data indicated that the first node of Ranvier is not the site of AP initiation in layer 5 pyramidal neurons. Finally, we observed that the site of AP initiation was similar during AP generation via synaptic stimulation, as well as during brief and steady-state somatic current injection, after axonal inhibition (Fig. 7), and at different stages of development (Fig. 8). Together, these experiments indicate that the site of AP initiation in cortical layer 5 pyramidal neurons is robust under a variety of conditions.

The most likely explanation for the observed difference in the site of AP initiation in cortical layer 5 pyramidal neurons and cerebellar Purkinje neurons is that it is because of differences in cell morphology and channel distributions. Purkinje cells are known to have a very thin axon and a short initial segment (Clark et al., 2005). In addition, Purkinje cells have an extensive dendritic tree, which lacks voltage-activated sodium channels, and is likely to exert a significantly greater electrical load on the soma than is the case in layer 5 pyramidal neurons. Together, these anatomical features would be expected to "push" the site of AP initiation further down the axon in cerebellar Purkinje neurons.

\section{Why the initial segment?}

Historically, the lower AP threshold in the axon has been attributed to a high density of voltage-activated sodium channels in the axon initial segment. Support for this idea came from early electronmicroscope (EM) work, which showed that the initial segment has an electron dense undercoating that resembles that observed at nodes of Ranvier (Palay et al., 1968; Sloper and Powell, 1979). More recent studies using both nonspecific and subunit specific sodium channel antibodies also provide evidence for a high density of voltage-activated sodium channels in the initial segment of many neuronal types (Wollner and Catterall, 1986; Jenkins and Bennett, 2001; Komada and Soriano, 2002; Boiko et al., 2003). Our finding of AP initiation in the axon initial segment, as well as the experiments describing the impact of lowsodium applications and axon length on AP threshold, is consistent with these studies and indicates that the axon initial segment is highly excitable. Recent patch recordings, however, suggest that the initial segment contains a similar density of sodium channels to the soma (Colbert and Johnston, 1996; Colbert and Pan, 2002; Ruben et al., 2003). How can these findings be reconciled? One possibility is that there is an undiscovered region of high sodium channel density in the distal axon initial segment. This seems unlikely, however, because the patch recordings of Colbert and Pan (2002) were made over the entire initial segment region (up to $45 \mu \mathrm{m}$ from the soma). Alternatively, there is evidence that the properties rather than the density of sodium channels in the axon initial segment are different from sodium channels at the soma (Colbert and Pan, 2002). A third possibility is that the EM and antibody studies are accurate, and that the patch experiments underestimate the density of sodium channels in the axon initial segment, possibly because of an inability to detach sodium channels in the axon initial segment from the cytoskeleton (Ruben et al., 2003).

Our finding of AP initiation in the distal axon initial segment is consistent with theoretical studies, which predict that under conditions of a highly excitable initial segment, its distal end is the favored site for AP initiation (Mainen et al., 1995). Presumably, this is the case, because this region is maximally shielded from the large electrical load of the soma and dendrites while being adjacent to the myelinated axon, which would be expected to have a high membrane resistance and low capacitance.

Once initiated, APs propagate both orthodromically down the axon to cause transmitter release onto postsynaptic neurons, as well as antidromically back into the soma and dendrites (Stuart et al., 1997b). Initiation of APs in the axon initial segment rather than more distal axonal sites such as the first nodes of Ranvier would be expected to increase the probability of robust AP backpropagation, while at the same time reducing the distance excitatory synaptic inputs need to propagate to influence AP initiation. This may be important for reducing cable filtering of synaptic events, thereby allowing their temporal characteristics to influence AP initiation. The central location of the axon, which usually emerges from the soma of neurons, is ideally positioned to respond to all synaptic inputs a neuron receives. Although the soma could also serve this purpose, its large surface area, as well as proximity to large dendritic branches, makes it an electrically unfavorable location. This disadvantage could be surmounted if the density of somatic sodium channels was sufficiently high, but because of the large number of sodium channels that this would require, this is an energetically unfavorable solution. Finally, it is important to note that the axon initial segment receives direct inhibitory synaptic input from GABAergic interneurons (DeFelipe et al., 1985; Buhl et al., 1994). These inhibitory synapses are located directly at the site of AP initiation and thus are ideally placed to inhibit the generation of APs.

In conclusion, although it has long been recognized that APs are initiated in the axon, exactly where in the axon has been the subject of much debate. We show here that APs in cortical layer 5 pyramidal neurons are initiated at the distal end of the axon initial segment $\sim 35 \mu \mathrm{m}$ from the soma. Once initiated, the AP propagates along the axon in a saltatory manor. These findings have important implications for understanding how single neurons translate synaptic inputs into an output signal, the action potential.

\section{References}

Antic SD (2003) Action potentials in basal and oblique dendrites of rat neocortical pyramidal neurons. J Physiol (Lond) 550:35-50.

Bishop PO, Levick WR (1956) Saltatory conduction in single isolated and non-isolated myelinated nerve fibres. J Cell Physiol 48:1-34.

Boiko T, Rasband MN, Levinson SR, Caldwell JH, Mandel G, Trimmer JS, Matthews G (2001) Compact myelin dictates the differential targeting of two sodium channel isoforms in the same axon. Neuron 30:91-104.

Boiko T, Van Wart A, Caldwell JH, Levinson SR, Trimmer JS, Matthews G (2003) Functional specialization of the axon initial segment by isoformspecific sodium channel targeting. J Neurosci 23:2306-2313.

Buhl EH, Han ZS, Lorinczi Z, Stezhka VV, Karnup SV, Somogyi P (1994) Physiological properties of anatomically identified axo-axonic cells in the rat hippocampus. J Neurophysiol 71:1289-1307.

Clark BA, Monsivais P, Branco T, London M, Hausser M (2005) The site of action potential initiation in cerebellar Purkinje neurons. Nat Neurosci 8:137-139.

Colbert CM, Johnston D (1996) Axonal action-potential initiation and $\mathrm{Na}^{+}$channel densities in the soma and axon initial segment of subicular pyramidal neurons. J Neurosci 16:6676-6686. 
Colbert CM, Pan E (2002) Ion channel properties underlying axonal action potential initiation in pyramidal neurons. Nat Neurosci 5:533-538.

Coombs JS, Curtis DR, Eccles JC (1957) The interpretation of spike potentials of motoneurones. J Physiol (Lond) 139:198-231.

DeFelipe J, Hendry SH, Jones EG, Schmechel D (1985) Variability in the terminations of GABAergic chandelier cell axons on initial segments of pyramidal cell axons in the monkey sensory-motor cortex. J Comp Neurol 231:364-384.

Dodge FA, Cooley JW (1973) Action potential of the motoneuron. IBM J Res Dev 17:219-229.

Fatt P (1957) Sequence of events in synaptic activation of a motoneurone. J Neurophysiol 20:61-80.

Fraher JP, Kaar GF (1984) The transitional node of Ranvier at the junction of the central and peripheral nervous systems: an ultrastructural study of its development and mature form. J Anat 139:215-238.

Freund TF, Martin KA, Smith AD, Somogyi P (1983) Glutamate decarboxylase-immunoreactive terminals of Golgi-impregnated axoaxonic cells and of presumed basket cells in synaptic contact with pyramidal neurons of the cat's visual cortex. J Comp Neurol 221:263-278.

Fuortes MGF, Frank K, Becker MC (1957) Steps in the production of motoneuron spikes. J Gen Physiol 40:735-752.

Gogan P, Gueritaud JP, Tyc-Dumont S (1983) Comparison of antidromic and orthodromic action potentials of identified motor axons in the cat's brain stem. J Physiol (Lond) 335:205-220.

Huxley AF, Stampfli R (1949) Evidence for saltatory conduction in peripheral myelinated nerve fibres. J Physiol (Lond) 108:315-339.

Jenkins SM, Bennett V (2001) Ankyrin-G coordinates assembly of the spectrin-based membrane skeleton, voltage-gated sodium channels, and L1 CAMs at Purkinje neuron initial segments. J Cell Biol 155:739-746.

Komada M, Soriano P (2002) $\beta$ IV-spectrin regulates sodium channel clus- tering through ankyrin-G at axon initial segments and nodes of Ranvier. J Cell Biol 156:337-348.

Mainen ZF, Joerges J, Huguenard JR, Sejnowski TJ (1995) A model of spike initiation in neocortical pyramidal neurons. Neuron 15:1427-1439.

Palay SL, Sotelo C, Peters A, Orkand PM (1968) The axon hillock and the initial segment. J Cell Biol 38:193-201.

Peters A, Proskauer CC, Ribak CE (1982) Chandelier cells in rat visual cortex. J Comp Neurol 206:397-416.

Ruben PC, Ilschner SU, Williams SR, Stuart GJ (2003) Distribution and properties of sodium channels in the axon initial segment of layer 5 cortical pyramidal neurons. Soc Neurosci Abstr 33:476.2.

Sloper JJ, Powell TP (1979) A study of the axon initial segment and proximal axon of neurons in the primate motor and somatic sensory cortices. Philos Trans R Soc Lond B Biol Sci 285:173-197.

Stuart G, Häusser M (1994) Initiation and spread of sodium action potentials in cerebellar Purkinje cells. Neuron 13:703-712.

Stuart G, Schiller J, Sakmann B (1997a) Action potential initiation and propagation in rat neocortical pyramidal neurons. J Physiol (Lond) 505:617-632.

Stuart G, Spruston N, Sakmann B, Häusser M (1997b) Action potential initiation and backpropagation in neurons of the mammalian central nervous system. Trends Neurosci 20:125-131.

Stuart GJ, Sakmann B (1994) Active propagation of somatic action potentials into neocortical pyramidal cell dendrites. Nature 367:69-72.

Stuart GJ, Dodt H-U, Sakmann B (1993) Patch-clamp recordings from the soma and dendrites of neurones in brain slices using infrared video microscopy. Pflügers Arch 423:511-518.

Wollner DA, Catterall WA (1986) Localization of sodium channels in axon hillocks and initial segments of retinal ganglion cells. Proc Natl Acad Sci USA 83:8424-8428. 